\title{
Molecular Dynamics Study on Melting Phenomena in Cu-Ag Eutectic System
}

\author{
Takahiro ITO** \\ **Department of Mechanical Engineering, Toyohashi University of Technology, \\ 1-1 Hibarigaoka, Tempaku-cho, Toyohashi, Aichi 441-8580, Japan \\ E-mail: takaito@mech.tut.ac.jp
}

\begin{abstract}
This study is made to elucidate the primary mechanism dominating the inception and progress of eutectic melting between two solid metals contacting with each other. In this study, $\mathrm{Cu}-\mathrm{Ag}$ eutectic system is simulated with classical molecular dynamics using Embedded Atom Method ${ }^{(1)}$. First, melting temperature of solid solution of $\mathrm{Cu}-\mathrm{Ag}$ binary system is investigated. The melting temperature depends on the atomic concentration of $\mathrm{Cu}$ and follows the liquidus line obtained in the experiments. The minimum melting temperature was obtained at the eutectic concentration. The melting behavior on the interface between two pure $\mathrm{Cu}$ and $\mathrm{Ag}$ slabs are then simulated. The mutual diffusion at the interface was considerably enhanced by the surface melting of both the metals. It is shown that the melting temperature at the interface is lowered depending on the local value of the atomic fraction and is almost identical to that of the solid solution with the corresponding atomic fraction.
\end{abstract}

Key words: Molecular Dynamics, Eutectic, Melting, Atomic Diffusion, Embedded Atom Method

\section{Introduction}

Eutectic phenomenon is one of the important interactions between two or more different metals in contact with each other. The melting point of eutectic alloy (eutectic point) is considerably lower than either of the individual components, and the melting point at the interface between pure solids of the corresponding metals is also lowered to the eutectic temperature.

This phenomenon has been widely utilized in a lot of metallurgy processes, alloy producing, and so on; it may, however, also cause unanticipated situation on some engineering processes. In the case of nuclear reactor engineering, especially for sodium-cooled fast reactor, such decrease in the melting point of the metal fuel or structure metal (e.g. in Pu-Fe eutectic system) would deteriorate the system integrity and bring remarkable influence on the progress of core disruptive accident. For exact prediction or estimation of such reactor event, it is thus necessary to analyze the phase transition process including eutectic reaction, in high accuracy and resolution. Since advancing of melting are strongly affected by the local condition of the material, the establishment of the analysis technique for such purpose requires understanding of eutectic melting phenomena from a microscopic point of view. ${ }^{(2)}$ Molecular dynamics (MD) is a powerful tool to analyze microscopic, atomic behavior in such interaction in eutectic systems.

$\mathrm{Cu}-\mathrm{Ag}$ eutectic system has been widely well analyzed by MD simulation under its wide application in practice use, and many investigations have been performed with a focus on eutectic melting and corresponding phenomena. Chen et al. ${ }^{(3)}$ have investigated the dependence of thickening speed of mixing region on the pressure normal to the interface

*Received 1 Aug., 2008 (No. 08-0561) [DOI: 10.1299/jpes.3.261] 
between $\mathrm{Cu}$ and $\mathrm{Ag}$ plates. The interphase semicoherent boundary of $\mathrm{Cu}-\mathrm{Ag}$ system was calculated by Roger III et al. ${ }^{(4)}$ and they have shown that the phase diagram can be drawn using MD with fairly good agreement with the experiments. Webb III et al. ${ }^{(5)}$ estimated the eutectic point in $\mathrm{Cu}-\mathrm{Ag}$ and obtained good consistency with experiments. They also analyzed intrusion phenomena of liquid $\mathrm{Ag}$ into solid $\mathrm{Cu}$ plate by eutectic melting.

However, primary mechanism dominating the advancing of melting interface between two solid metals composing eutectic system has been still unclear. This study is made to understand microscopic mechanisms on the inception and progress of melting at the solid-solid interface of two metals using $\mathrm{Cu}-\mathrm{Ag}$ eutectic system for which the potential model of MD has been well developed. The relation between the atomic distribution and magnitude of atomic diffusion in the interface region are estimated under several temperature conditions. To elucidate the effect of atomic composition on the melting temperature at the interface, the melting behavior at the interface is compared with that in the solid solution. These understandings would be useful to choosing parameters to be referred to for the development of the potential model for other eutectic systems.

In Sec. 2 analysis method is briefly described. Section 3 provides analysis results on the melting point of the $\mathrm{Cu}-\mathrm{Ag}$ solid solution. The melting behavior on the interface between two pure materials is also shown. The position of the melting surface (liquid-solid interface) determined based on the mobility of atoms is related to the atomic fraction there. Section 4 gives a summary of this paper.

\section{Analysis Method}

To simulate the atomic behavior of eutectic metals by classical molecular dynamics, Embedded Atom Method (EAM) ${ }^{(1)}$ has been used as the potential model. The EAM potential has been often used to simulate atomic motion of metals, properties of metals, and also applied to the analyses of eutectic phenomena. ${ }^{(3-6)}$ Using the EAM potential, the total energy of atoms is represented as

$$
E_{\text {tot }}=\sum_{i} F_{i}\left(\rho_{h, i}\right)+\frac{1}{2} \sum_{i} \sum_{j \neq i i} \phi_{i j}\left(R_{i j}\right),
$$

where $F_{i}(\rho)$ is the energy to embed atom $i$ into the background electron density $\rho, \rho h, i$ the host electron density at atom $i$ and $\phi_{i j}\left(R_{i j}\right)$ represents the core-core pair repulsion between atoms $i$ and $j$ separated by the distance $R_{i j}$. The electron density $\rho_{h, i}$ is approximated by the superposition of atomic densities as

$$
\rho_{h, i}=\sum_{j} \rho_{j}^{a}\left(R_{i j}\right),
$$

where $\rho_{j}^{a}\left(R_{i j}\right)$ is the contribution of electron density of atom $j$, which can be taken from the calculations $^{(7,8)}$ based on Hartree-Fock theory.

The functional form of $\phi$ is based on the Coulomb interaction, written as

$$
\phi_{i j}=Z_{i}\left(R_{i j}\right) Z_{j}\left(R_{i j}\right) / R_{i j} \text {. }
$$

In this equation, $Z_{i}$ and $Z_{j}$ are the effective charges of atoms $i$ and $j$, respectively, and are assumed to have a simple parameterized form of

$$
Z(r)=Z_{0}\left(1+\beta r^{v}\right) \exp (-\alpha r)
$$

where $Z_{0}$ is the number of outer electrons of the atom and $\beta, v, \alpha$ are the coefficients empirically determined.

The function $F$ is determined through the sublimation energy. Rose et al. ${ }^{(9)}$ have shown that the sublimation energy and the lattice constant is scaled to a simple universal function,

$$
E(a)=-E_{\text {sub }}\left(1+a^{*}\right) \exp \left(-a^{*}\right) .
$$

In this expression, $E_{\text {sub }}$ represents the absolute value of sublimation energy at zero-temperature and -pressure, $a^{*}$ is a quantity representing the deviation from the equilibrium lattice constant, 


$$
a^{*}=\left(a / a_{0}-1\right)\left(E_{\text {sub }} / 9 B \Omega\right)^{1 / 2},
$$

where $a_{0}$ and $a$ are the lattice constant under the equilibrium and concerning conditions, respectively, $B$ is the bulk modulus and $\Omega$ the equilibrium volume per atom. The specific representations of $F, Z$ and the other parameters for $\mathrm{Cu}$ and $\mathrm{Ag}$ have been empirically determined by Foiles et al. ${ }^{(6)}$ using the experimental data of elastic constants, vacancy-formation energy and so on.

The time step is set to be $1 \mathrm{fs}$ through all the simulations.

\section{Results and Discussions}

\subsection{Melting point of solid solution}

At the interface between $\mathrm{Ag}$ and $\mathrm{Cu}$ solid slabs, $\mathrm{Ag}$ and $\mathrm{Cu}$ atoms mutually diffuse and compose a mixture phase, which corresponds to solid solution if the atoms there are in solid state. Since the state of this solid solution apparently dominates the melting phenomena there, the melting point $T_{m}$ of $\mathrm{Cu}-\mathrm{Ag}$ solid solution was then firstly investigated. In normal cooling condition, liquid alloy of $\mathrm{Cu}$ and $\mathrm{Ag}$ separates to $\mathrm{Cu}$-rich and

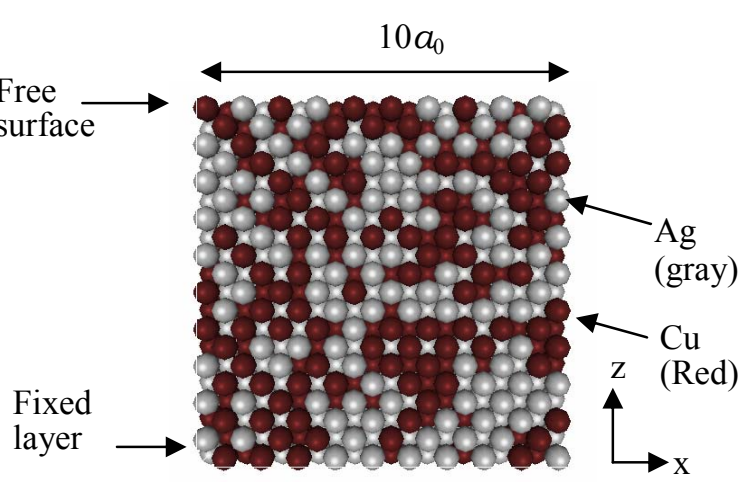

Fig. 1 Calculational system for the investigation of melting point of solid solution. The origin of the $z$ axis is taken at the elevation of the bottom layer.

Ag-rich phases because of the limitation in the solubility between them; however, rapid quenching of the liquid alloy generates solid solution for all range of $\mathrm{Cu}$ concentration. ${ }^{(10)}$

Melting point is investigated by simulating a semi-infinite plate of $\mathrm{Cu}-\mathrm{Ag}$ solid solution accompanied with free surface. On the free surface, melting takes place even when the temperature is lower than its melting point, which is termed surface melting. ${ }^{(1)}$ The surface melting is caused by the fact that on/near the surface the force constraining the atoms at their own lattice sites is weaker than that in the bulk of the plate. When the temperature $T$ is lower than $T_{m}$, only the atoms near the surface are able to leave their lattice positions and move around. On the other hand, for $T>T_{m}$, the melting process develops from the free surface into the bulk of the solid solution.

Characteristic atomic distribution at the initial state is depicted in Fig. 1. At first $\mathrm{Cu}$ atoms are located on the face-centered-cubic (fcc) lattice and then some atoms are randomly replaced by $\mathrm{Ag}$ atoms so as to make the number of $\mathrm{Cu}$ atoms correspond to the target value of number concentration of $\mathrm{Cu}\left(X_{C u}\right)$. The total number of atoms is 4000 .

The lattice size is decided by preliminary calculations with NPT ensemble, where the pressure is kept at zero by Berendsen barostat ${ }^{(12)}$ and the temperature maintained to each target value by Nosé thermostat ${ }^{(13)}$. The periodic boundary condition is imposed on the $x$ and $y$-direction. The cell sizes in both the $x$ - and $y$-directions are set to be 10 times of the lattice constant $a_{0}$ and the initial thickness of the slab also to be $10 a_{0}$. The calculational results shown here are not affected by the change in the cell size at least up to $15 a_{0}$.

The atoms which compose the bottom layer are constrained to be fixed at their initial lattice position to keep the bottom surface planar. On the other hand, the atoms on the top surface are not subject to any particular constraining forces, that is, the atoms on the top surface form an actual free surface.

The melting point is estimated by investigating the time evolution of the temporal diffusion coefficient defined as, 


$$
d(z)=\frac{1}{6 \Delta t N_{z}(z)} \sum_{i}\left(\boldsymbol{r}_{i}(t+\Delta t)-\boldsymbol{r}_{\boldsymbol{i}}(t)\right)^{2} .
$$

with $N_{z}(z)$ the number of atoms in a layer with thickness of $0.025 \mathrm{~nm}$ at elevation $z$. In the present case $\Delta t$ is set at $2 \mathrm{ps}$ in order to obtain $d(z)$ with sufficient time resolution. Such a short time interval results in $d(z)$ dependent on $\Delta t$; however $\Delta t$ of $2 \mathrm{ps}$, as shown later, gives clear difference between $d(z)$ for liquid and solid states. In order to remove poor statistical results, $d(z)$ is defined as zero when $N_{z}(z)$ is less than 5 .

Shown in Fig. 2(a) and (b) are the instantaneous distribution of $d(\mathrm{z})$ of $\mathrm{Ag}$ in solid solution with $40 \%$ of $X_{C u}$ for $850 \mathrm{~K}$ and $900 \mathrm{~K}$, respectively. For $T=850 \mathrm{~K}$, the distribution is almost unchanged against time. The value of $d(\mathrm{z})$ in $z<2.5 \mathrm{~nm}$ varies periodically and takes zero with an interval of about $0.20 \mathrm{~nm}$, which is almost identical to the half of the lattice constant observed in this condition. Since $d(z)=0$ represents that few atoms locate at $z$, such periodicity in $d(z)$ indicates the atoms in this region still form layered structure associated with the lattice structure. The peak value of $d(z)$ in this region is no more than $0.4 \times 10^{-9} \mathrm{~m}^{2} / \mathrm{s}$. Meanwhile, $d(z)$ in $z>2.5 \mathrm{~nm}$ is almost continuous and takes larger values than $1 \times 10^{-9} \mathrm{~m}^{2} / \mathrm{s}$, which means that the atoms move around with larger mobility than those in $z<2.5 \mathrm{~nm}$ and the lattice structure is no longer maintained. These results mean that the atoms which locate $z>2.5 \mathrm{~nm}$ behave as liquid state and the melting surface (solid-liquid interface) locates at $Z \approx 2.5 \mathrm{~nm}$.

Similar distributions can be seen for $T=900 \mathrm{~K}$ at $t=40$ ps. At $t=92$ ps for that temperature, the region in which $d(z)$ takes higher value is spreading downward and the melting surface descends to $Z \approx 1 \mathrm{~nm}$, resulting in the liquid thickness of 3 $\mathrm{nm}$.

The total mobility of the atoms in the concerning area can be obtained by $\int d(z) d z$. As shown in Fig. 2(c), $\int d(z) d z$ for $T=850 \mathrm{~K}$ gradually increases up to $1.8 \times 10^{-18} \mathrm{~m}^{3} / \mathrm{s}$ until $t=90 \mathrm{ps}$ and then becomes almost constant at that value. Conversely, in the case of $T=900 \quad \mathrm{~K}$, $\int d(z) d z$ increases monotonically with time.

The increase in $\int d(z) d z$ indicates the enhancement of the average mobility of each atom and/or the expansion of melting (liquid) area. Since $d(z)$ in the liquid region is almost constant at $1 \times 10^{-9} \mathrm{~m}^{2} / \mathrm{s}$ except for the vicinity of the top surface in the present case, increase in $\int d(z) d z$ with time represents thickening of the liquid region. The saturation of increase in $\int d(z) d z$ for $T=850 \mathrm{~K}$ then indicates that the melting is limited in a certain thickness from the surface, which is surface melting. On the contrary, continuous increase for $T=900 \mathrm{~K}$ represents that the temperature of $900 \mathrm{~K}$
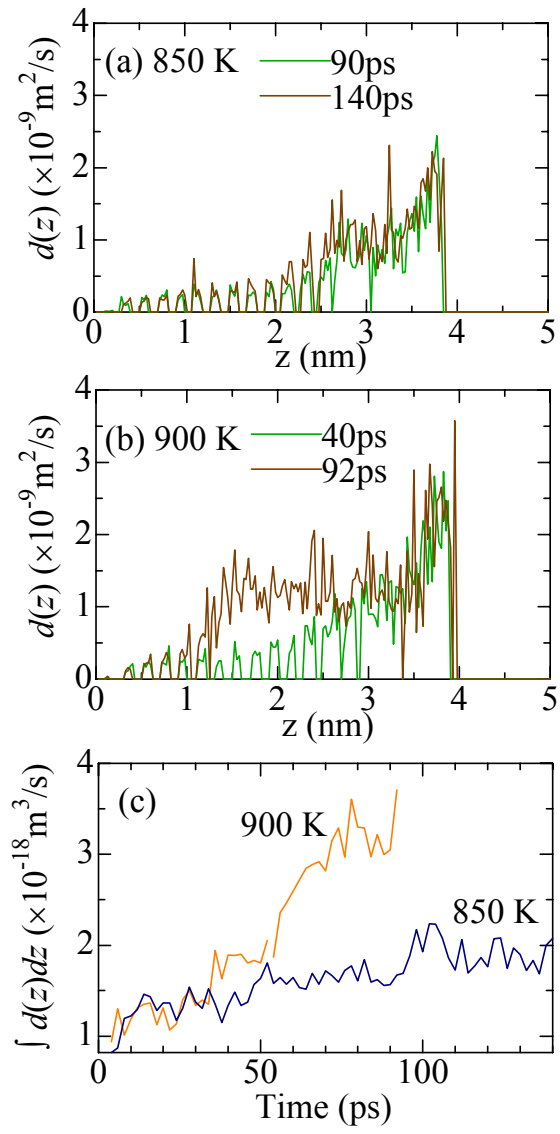

Fig. 2 (a) and (b) Instantaneous distribution of $d(z)$ for $T=850 \mathrm{~K}$ and $900 \mathrm{~K}$, respectively, and (c) time evolution of $\int d(z) d z$, all corresponding with $\mathrm{Ag}$ for $X_{\mathrm{Cu}}=40 \%$. 
exceeds the melting temperature. The calculational results for $T=875 \mathrm{~K}$ show almost the same behavior as those for $T=900 \mathrm{~K}, T_{m}$ is hence between $875 \mathrm{~K}$ and $850 \mathrm{~K}$ and can be approximately defined as the average value of them, $863 \mathrm{~K}$. The resolution of melting temperature obtained by this method is hence $25 \mathrm{~K}$.

Similar procedures were performed by changing $X_{\mathrm{Cu}}$ from zero to unity in order to obtain the dependence of $T_{m}$ on $X_{C u}$. The obtained values of $T_{m}$ are plotted in Fig. 3 against the atomic fraction of $\mathrm{Cu}, X_{C u}$. As shown in the figure, $T_{m}$ shows $\mathrm{V}$-shaped distributions as the liquidus lines in the $\mathrm{Cu}-\mathrm{Ag}$ phase diagram ${ }^{(14)}$ and takes a minimum value at $X_{\mathrm{Cu}}=0.41$. This concentration is consistent with the experimental value of eutectic point $\left(X_{C u}=0.399\right)$; however $T_{m}$ of $863 \mathrm{~K}$ at this concentration is lower than the experimental value of the eutectic temperature. One of the reasons for this discrepancy should be the fact that the analysis estimates the melting temperature for pure $\mathrm{Ag}$ as $1088 \mathrm{~K}$, which is lower than the experimental value of $1234 \mathrm{~K}^{(14)}$. Previous study by other researchers using EAM potential has also shown lower melting temperature of $\mathrm{Ag}^{(5)}$. The obtained value of $T_{m}$ for pure $\mathrm{Cu}$ is almost consistent with the experimental value of $1356 \mathrm{~K}^{(14)}$, similarly as the previous study with $\mathrm{EAM}^{(5)}$.

\subsection{Simulation of eutectic reaction between pure $\mathrm{Cu}$ and $\mathrm{Ag}$ slabs}

The simulation is performed using the system shown in Fig. 4, in which one surface of semi-infinite $\mathrm{Cu}$ plate is contacting with Ag plate. The upper side of the $\mathrm{Cu}$ plate is set to be free surface to keep the pressure in z-direction to be zero. The atoms in the bottom layer of Ag plate are constrained at the lattice sites in the equilibrium state in order to keep the two plates to be planar. The cell sizes in the $x$ - and $y$-direction, termed $L_{x}$ and $L_{y}$, respectively, are determined so as to minimize the discrepancy of the lattice size from those in the equilibrium state. In the present case we choose $L_{x}=L_{y}=5.7876 \mathrm{~nm}$, almost equivalent to $16 a_{0}(\mathrm{Cu})$ and $14 a_{0}(\mathrm{Ag})$. By using these values of $L_{x}$ and $L_{y}$, the deviation of lattice size from the equilibrium condition is suppressed less than $0.3 \%$. The initial thicknesses of both the $\mathrm{Cu}$ and $\mathrm{Ag}$ slabs are set to $15 a_{0}$. The simulation is performed with a constant temperature at $1050 \mathrm{~K}$ and the cell size is also kept constant.

In Figs. 5(a) and (b), projections of the atomic distributions onto $x-z$ plane are depicted for $t=1000 \mathrm{ps}$ and $3000 \mathrm{ps}$, respectively. It can be seen that, near the interface between $\mathrm{Cu}-\mathrm{Ag}$, the atoms leave their lattice sites and then mutual diffusion and melting are developing.

As the temperature $(1050 \mathrm{~K})$ is lower than the melting point of $\mathrm{Cu}$, melting for the $\mathrm{Cu}$ plate is seen only within thin region close to the top surface and the bulk of the $\mathrm{Cu}$ plate remains solid state.

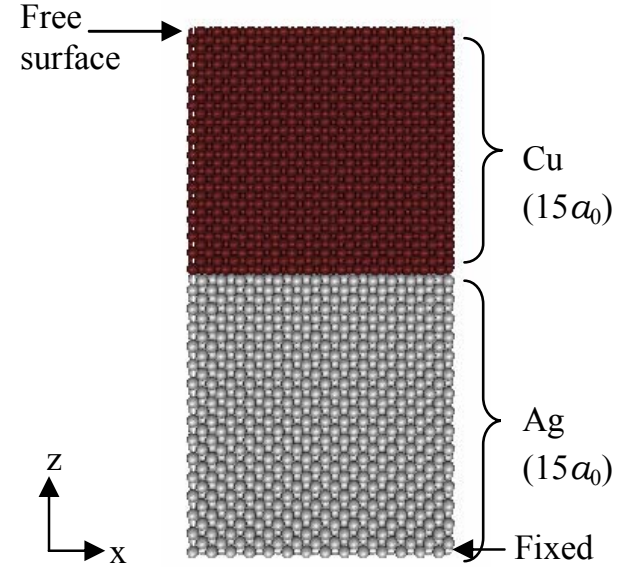

Fig. 4 System for the simulation of eutectic melting between pure $\mathrm{Cu}$ and $\mathrm{Ag}$ slabs. The origin of $\mathrm{z}$ axis is taken at the bottom layer. 


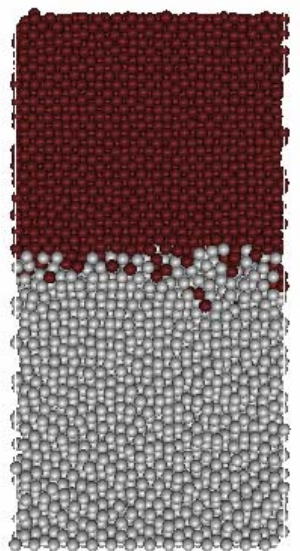

(a) $1000 \mathrm{ps}$

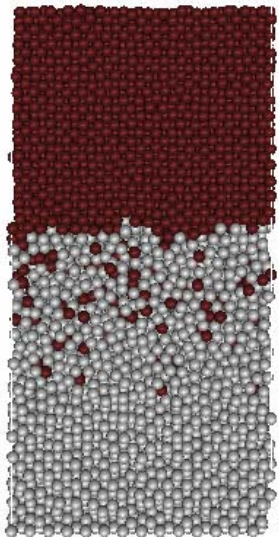

(b) $3000 \mathrm{ps}$

Fig. 5 Distributions of atoms $(T=1050 \mathrm{~K})$

The diffusion and melting at the interface is thus regarded to be introduced by the eutectic melting.

As shown in Fig. 6, $\int d(z) d z$ for both $\mathrm{Ag}$ and $\mathrm{Cu}$ are increasing with time, indicating that the melting region continues to thicken. Figure 6 also exhibits that the increasing rate of $\int d(z) d z$, i.e. $(d / d t) \int d(z) d z$, is not constant; the rate for $t>1500$ ps is larger than that for $t<1500$ ps. The reason of this difference will be discussed below.

Figures 7 and 8 depict the distributions of $d(z)$ and $X_{C u}$ at $t=2$ and $3000 \mathrm{ps}$, respectively. At $t=2 \mathrm{ps}, d(z)$ is approximately $0.3 \times 10^{-9} \mathrm{~m}^{2} / \mathrm{s}$ for $\mathrm{Cu}$ and $0.4 \times 10^{-9} \mathrm{~m}^{2} / \mathrm{s}$ for $\mathrm{Ag}$, except the vicinity of the interface $(z=6.2 \mathrm{~nm})$ and the top surface. As the $\mathrm{Cu}$ atoms have hardly diffused yet at this time, $X_{C u}$ varies steeply from 0.1 to unity at the interface as shown in Fig. 7(a). The large value of $d(z)$ near the interface $(z=7 \mathrm{~nm}$ in Fig. 7(b)) indicates that surface melting phenomena takes place there as well as at the top surface of $\mathrm{Cu}(z=12.5 \mathrm{~nm})$. In the present case, the equilibrium lattice constant of $\mathrm{Ag}$ differs from that of $\mathrm{Cu}$ by $13 \%$, hence the distribution of the potential wells associated with the $\mathrm{Cu}$ atoms is out of synchronization with the lattice sites of the $\mathrm{Ag}$ atoms, and vise versa. This enhances the possibility of the atoms to leave their lattice sites, resulting in that the atoms near the interface tend to melt as observed near the free surface. This surface melting observed in both the metals at the contacting region apparently enhances the mutual

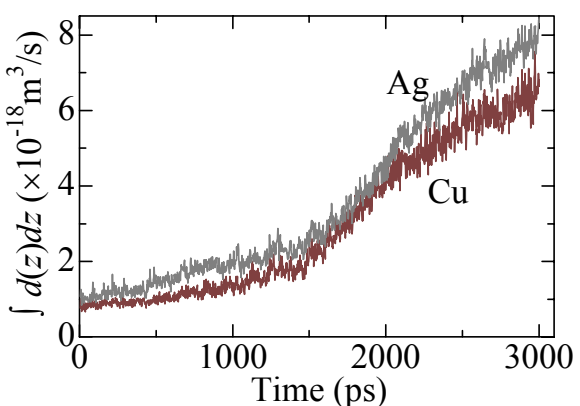

Fig. 6 Time trend of $\int d(z) d z$ in the simulation of eutectic reaction between pure slabs. $(T=1050 \mathrm{~K})$

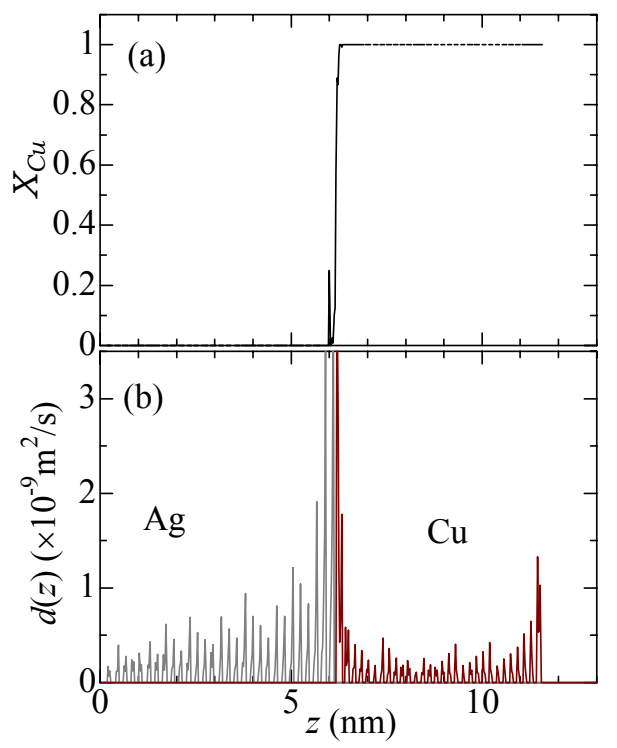

Fig. 7 Distribution of $X_{C u}($ a) and $d(z)$ (b) (at 2 ps ). 
diffusion at the interface to considerably higher extent as compared with the solid-state diffusion.

At $t=3000$ ps (Fig. 8), $X_{\mathrm{Cu}}$ gradually increases from zero to 0.4 for $3 \mathrm{~nm}<z<7$ $\mathrm{nm}$ where $d(z)$ for both $\mathrm{Cu}$ and $\mathrm{Ag}$ are approximately $2.2 \times 10^{-9} \mathrm{~m}^{2} / \mathrm{s}$. Around $z \sim 7$ $\mathrm{nm} X_{C u}$ steeply approaches to unity. The value of $d(z)$ in the region where $X_{C u}$ is zero or unity is less than $0.4 \times 10^{-9} \mathrm{~m}^{2} / \mathrm{s}$ and shows periodic distribution, which is identical to that of the bulk area of $\mathrm{Ag}$ or $\mathrm{Cu}$ at $t=2 \mathrm{ps}$. These distributions in $d(z)$ and $X_{C u}$ mean that $\mathrm{Cu}$ and $\mathrm{Ag}$ atoms in $3 \mathrm{~nm}<z<7 \mathrm{~nm}$ are mutually diffusing and behave as liquid and in other region they remain to be in solid state and are not melting.

Here let define melting surface as a plane at the elevation at which $d(z)$ takes $1.2 \times 10^{-9} \mathrm{~m}^{2} / \mathrm{s}$ which is the average value between liquid region $\left(d \approx 2.2 \times 10^{-9} \mathrm{~m}^{2} / \mathrm{s}\right)$ and solid region (approximately $0.3 \times 10^{-9}$ $\mathrm{m}^{2} / \mathrm{s}$ ). According to this criterion, the melting surfaces Fig. 8(b) are obtained as $z=3 \mathrm{~nm}$ and $6.7 \mathrm{~nm}$.

Shown in Fig. 9 are the time trends of the elevations of melting surfaces for $\mathrm{Ag}$ and $\mathrm{Cu}$ sides. The melting speed of $\mathrm{Cu}$ (i.e., the advancing speed of the melting surface for $\mathrm{Cu}$ rich side) is almost constant at $0.22 \mathrm{~m} / \mathrm{s}$ for whole simulation period. In contrast, that of Ag changes around $t=1500 \mathrm{ps}$. In $t<1500$ The change in the melting speed of $\mathrm{Ag}$ at $t=1500 \mathrm{ps}$ corresponds to the variation of the increasing rate of $\int d(z) d z$ as previously shown in Fig. 6. This variation in the melting speed of Ag surface can be related with the time variations in number density distributions of $\mathrm{Ag}$ and $\mathrm{Cu}$ atoms, shown in Fig. 10.

At $t=1400 \mathrm{ps}$, oscillation in the number density $\rho_{N}$ is observed for all the range of $z$, which represents formation of layered structure of atoms. Outside the liquid region, this layered structure corresponds to the crystalline structure of atoms. The layers formed in the liquid region is associated with that often observed near wall(solid)-liquid interface, where diffusion of atoms may be suppressed lower than in the bulk liquid. ${ }^{(15)}$

At $1700 \mathrm{ps}$ the layered structure in the center of liquid region are weakening and at last (1800 ps) almost disappears. The oscillation amplitude in the liquid region near the melting surface also decreases as compared with that at $1400 \mathrm{ps}$. This mitigation of the oscillation in the density distribution should increase the atomic diffusive motion in $z$ direction to the extent expected in bulk liquid, which promotes the melting at the melting surface. As a result, the advancing speed of the $\mathrm{Ag}$ interface, i.e. the increasing rate in $d(z)$, $(d / d t) \int d(z) d z$ (in Fig. 6), are raised.

Figure 10 also shows that $\rho_{N}$ for $\mathrm{Cu}$ takes smaller values than that for $\mathrm{Ag}$ in the liquid region and its oscillation amplitude decays faster than that of Ag. These behaviors indicate 
that the mutual diffusion at the interface is progressed mainly by penetration of $\mathrm{Cu}$ atoms into $\mathrm{Ag}$ layers and the interlayer motion of the $\mathrm{Cu}$ atoms grows more rapidly than the $\mathrm{Ag}$ atoms.

The observed progress of the diffusive motion of $\mathrm{Cu}$ in the MD simulation is as follows. Firstly, vacancy sites are formed in $\mathrm{Ag}$ side by running away of $\mathrm{Ag}$ atoms from their own sites, which is mainly induced by surface melting of Ag. Subsequently, $\mathrm{Cu}$ atoms in the next layer go into the vacancy sites.

Such diffusion of $\mathrm{Cu}$ atoms increases the concentration of $\mathrm{Cu}$ there, and leads to lowering of the melting temperature. Melting then should take place when the associated local melting temperature decreases to the actual temperature.

The local melting temperature is supposed to be same as $T_{m}$ of solid solution composed of $X_{C u}$ identical to that at the concerning location. In order to confirm this relation, the elevation of the melting surface is compared with that estimated from the distribution of $X_{C u}$ near the interface and the relation between $T_{m}$ and $X_{C u}$ for solid solution. For the present case $T=1050 \mathrm{~K}$ thus the concentration of solid solution which melt at this temperature is found from Fig. 3 as $X_{C u}=0.06$ or 0.7 .

Figure 11 shows the variation of the elevations where $X_{C u}$ is 0.06 or 0.7 in comparison with melting surfaces obtained by $d(z)$ shown in Fig. 9. They show good agreement. This result represents that the local melting temperature is lowered in accordance with the local concentration of $\mathrm{Cu}$. It is worth noting that, despite the steep increase in $X_{\mathrm{Cu}}$ around both the melting surfaces shown in Fig. 8, that is, non-negligible increase in $X_{C u}$ within the interval of the layer $(\approx 0.20 \mathrm{~nm})$, the melting temperature there is lowered, quantitatively following that observed in bulk solid solution of which $X_{C u}$ is spatially constant.

A similar simulation is made for $T=1200 \mathrm{~K}$. This temperature is higher than the melting temperature of pure $\mathrm{Ag}$, thus equilibrium state of liquid $\mathrm{Ag}$ is calculated in advance, and then the liquid $\mathrm{Ag}$ is put on the $\mathrm{Cu}$ slab. The top of the liquid $\mathrm{Ag}$ is free surface and atom positions in the bottom layer of $\mathrm{Cu}$ slab are fixed at each lattice site. The periodic boundary condition is imposed in the $x$ - and $y$-directions, as did in the case of $T=1050 \mathrm{~K}$. The cell sizes in the $x$ - and $y$-direction are taken equivalent to $23 a_{0}$ of $\mathrm{Cu}$ atoms. The initial thickness of $\mathrm{Cu}$ slab and $\mathrm{Ag}$ liquid is $10 a_{0}$, namely $3.616 \mathrm{~nm}$ and $4.41 \mathrm{~nm}$, respectively.

The atomic distributions for $t=2$ ps and 2700 ps are shown in Fig. 12. For $t=2700$ ps, it 


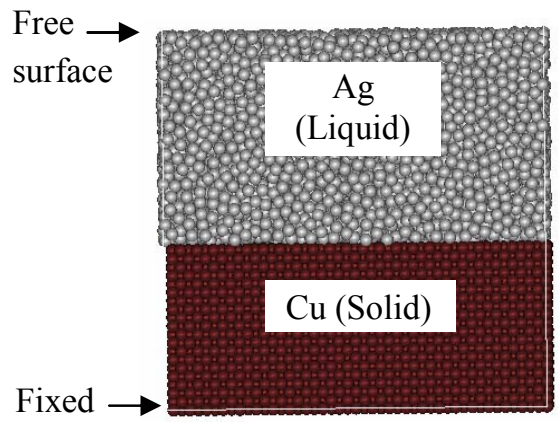

(a) 2 ps

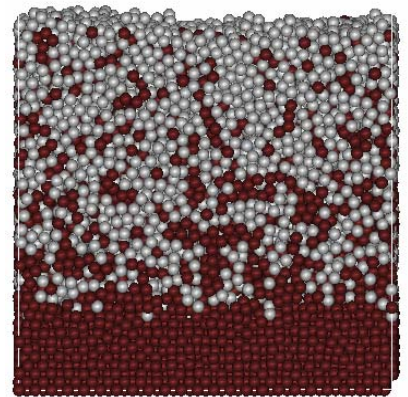

(b) $2700 \mathrm{ps}$

Fig. 12 Distributions of atoms ( $T=1200 \mathrm{~K}$ )

can be seen that $\mathrm{Cu}$ atoms dissolve and diffuse into liquid $\mathrm{Ag}$ and the melting surface locates at the lower elevation than that in $t=2$ ps.

The distribution of $X_{C u}$ and $d(z)$ of $\mathrm{Cu}$ are shown in Fig. 13 for $t=2$ and 2700 ps. At $t=2 \mathrm{ps}, X_{\mathrm{C} u}$ shows a steep variation from unity to zero at $z=3.8 \mathrm{~nm}$, indicating the diffusion of $\mathrm{Cu}$ has not developed yet. The value of $d(z)$ is approximately $0.3 \times 10^{-9} \mathrm{~m}^{2} / \mathrm{s}$ for $z<3 \mathrm{~nm}$, and exhibits higher value in the upper region. At 2700 ps, $X_{C u}$ decreases in $Z>1.8 \mathrm{~nm}$ and the region where $\mathrm{Cu}$ atoms are present (i.e. $X_{C u}>0$ ) is spreading upward. This represents the diffusion of $\mathrm{Cu}$ atoms into liquid $\mathrm{Ag}$ region. The value of $d(z)$ increases from $0.3 \times 10^{-9} \mathrm{~m}^{2} / \mathrm{s}$ to $3.0 \times 10^{-9}$ $\mathrm{m}^{2} / \mathrm{s}$ around $z=1.8 \mathrm{~nm}$ where $X_{C u}$ decreases from unity.

Figure 14 shows the time trend of the elevation of melting surface, determined by $d(z)$ and $X_{C u}$. The criterion of melting is $d=1.2 \times 10^{-9} \mathrm{~m}^{2} / \mathrm{s}$, same as used in Fig. 11. The elevation of the melting surface descends with time and its descending speed is $\sim 0.63 \mathrm{~m} / \mathrm{s}$, approximately 3 times of that of $\mathrm{Cu}$ side for $T=1050 \mathrm{~K}$ as shown in Fig.10. The red curve is drawn as the elevation where $X_{C u}=0.9$. This value is selected from the fact that solid solution of that $X_{C u}$, as known from Fig. 3, is supposed to melt at $1200 \mathrm{~K}$. As expected from the previous result shown in Fig. 11, these curves agree well with each other. This result also supports the argument that the local concentration of $\mathrm{Cu}$ dominates the melting temperature there.

\section{Summary}

Molecular dynamics study was performed to investigate the microscopic mechanism of

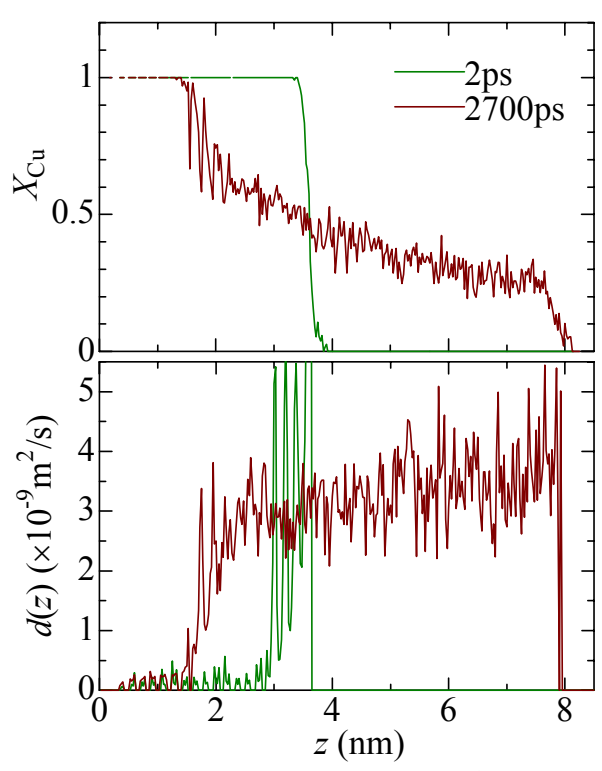

Fig. 13 Distribution of $X_{C u}$ and $d(z)$ of $\mathrm{Cu}$ $(T=1200 \mathrm{~K})$

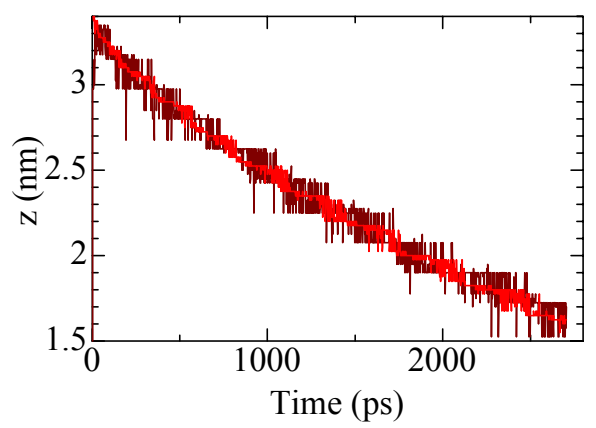

Fig. 14 Time trend of the elevation of the melting surface $(T=1200 \mathrm{~K})$. Brown: elevation based on $d(z)$, red:elevation where $X_{C u}$ is 0.9 . 
the initiation and evolution of eutectic reaction between two metals. Using the Embedded Atom Method $^{(1)}$ for the molecular dynamics calculation, melting behavior of $\mathrm{Cu}-\mathrm{Ag}$ solid solution and reaction at the interface between pure slabs of $\mathrm{Cu}$ and $\mathrm{Ag}$ were analyzed.

- The melting temperature of solid solution follows the liquidus line in the experimentally-obtained $\mathrm{Cu}-\mathrm{Ag}$ phase diagram ${ }^{(14)}$ and takes a minimum value of $863 \mathrm{~K}$ at the atomic fraction of $\mathrm{Cu}\left(X_{C u}\right)$ of 0.41 , almost in agreement with the eutectic composition in the phase diagram $\left(X_{C u}=0.399\right)$.

- When $\mathrm{Cu}$ and $\mathrm{Ag}$ solid plates are in contact with each other under the temperature of $1050 \mathrm{~K}$ which is higher than the eutectic temperature, surface melting at the $\mathrm{Cu}-\mathrm{Ag}$ contacting interface takes place before the progress of eutectic melting. This should enhance the mutual diffusion which consequently leads to the lowering of the melting temperature at the interface.

- While the liquid region in between $\mathrm{Cu}$ - and $\mathrm{Ag}$-side melting surfaces is fully structured by atomic layers parallel to the surface, the advancing speed of the melting surface is suppressed. This should be because that the interlayer diffusion in the layered structure is lower than in bulk liquid where such structure is not observed.

- Although at the interface $X_{C u}$ steeply varies in the direction normal to the interface, the melting temperature there is lowered depending on $X_{C u}$ from each of the pure materials, as seen in solid solution where $X_{C u}$ is almost spatially uniform.

\section{Acknowledgement}

This study was carried out within the task "R \& D of the Next Generation Safety Analysis Methods for Fast Reactors with New Computational Science and Technology" entrusted to University of Tokyo by the Ministry of Education, Culture, Sports, Science and Technology of Japan (MEXT).

\section{References}

(1) Daw, M. S. and Baskes, M. I., "Embedded-atom method: Derivation and application to impurities, surfaces, and other defects in metals", Phys. Rev. B 29 (1984) pp6443-6453.

(2) Koshizuka, S., Liu, J., Shirakawa, N., Uehara, Y., Naitoh, M., Yamamoto, Y., "R\&D of the Next Generation Safety Analysis Methods for Fast Reactors with New Computational Science and Technology (1) Brief Introduction of the Project and Development of Structural Mechanics Module of the COMPASS Code," Proceedings of the 16th International Conference on Nuclear Engineering (ICONE16), Orlando, Florida, USA (May 11-15, 2008) ICONE16-48499.

(3) Chen, S. D., Soh, A. K. and Ke, H. J., "Molecular dynamics modeling of diffusion bonding", Scripta Mater. Vol. 52 (2005), 1135-1140.

(4) Roger III, J. P., Wynblatt, P., Foiles, S. M. and Baskes, M. I., "Monte Carlo Simulation of the Cu-Ag (001) Semicoherent Interphase Boundary”, Acta Metall. Mater. Vol. 38 (1990), pp177-184.

(5) Webb III, E. B., Gary, S. G., Heine, D. R. and hoyt, J. J., "Dissolutive Wetting of Ag on Cu: A Molecular Dynamics Simulation Study”, Acta Meter. Vol. 53 (2005), pp3163-3177.

(6) Foiles, S. M., Baskes, M. I. and Daw, M. S., "Embedded-Atom-Method Functions for the FCC Metals Cu, Ag, Au, Ni, Pd, Pt and Their Alloys", Phys. Rev. B Vol. 33 (1986), pp7983-7991.

(7) Clementi, E. and Roetti, C., "Roothaan-Hartree-Fock Atomic Wavefunctions Basic Function and Their Coefiicients for Ground and Certain Excited States of Neutral and ionized Atoms, Z $\leq 54$ ”, At. Data Nucl. Data Tables Vol. 14 (1974), pp177-478.

(8) McLean, A. D. and McLean, R. S., "Roothaan-Hartree-Fock Atomic Wavefunctions Slater Basis-Set Expansions for Z=55-92”, ibid. Vol. 26 (1981), 197.

(9) Rose., J. H., Smith, J. R., Guinea, F. and Ferrante, J., "Universal features of the equation of 
state of metals", Phys. Rev. B Vol. 29 (1984), pp2963-2969.

(10) Sheng, H.W., Widle, G. and Ma, E., "The competing crystalline and amorphous solid solutions in the Ag-Cu system", Acta Mater. Vol. 50 (2002) pp475-488.

(11) Tartaglino, U. and Tosatti, E., "Strain Effects at Solid Surfaces near the Melting Point", Surf. Sci. Vol.532-535 (2003), pp623-627.

(12) Berendsen, H. J. C., Postma, J. P. M., van Gunsteren, W. F., DiNola, A. and Haak, J. R., "Molecular Dynamics with Coupling to an External Bath", J. Chem. Phys. Vol. 81 (1984), pp3684-3690.

(13) Nosé, S., "A Unified Formulation of the Constant Temperature molecular Dynamics Methods", J. Chem. Phys. Vol. 81 (1984), pp511-519 (1984).

(14) e.g., ASM International. Handbook Committee, ASM Handbook Vol. 3 Alloy Phase Diagrams, ASM International (1992).

(15) Thomas, J. A. and McGaughey, A. J. H., "Effect of Surface Wettability on Liquid Density, Structure, and Diffusion near a Solid Surface”, J. Chem. Phys. Vol. 126 (2007), p.034707. 